\title{
Galois Covers of Degree $p$ and Semi-Stable Reduction of Curves in Mixed Characteristics
}

By

\author{
Mohamed SAÏDI*
}

\begin{abstract}
In this paper we study the degeneration of a Galois cover $f: \mathcal{Y} \rightarrow \mathcal{X}$ of degree $p$ above a proper and smooth curve $\mathcal{X}$ over a complete discrete valuation ring of mixed characteristics $(0, p)$. We exhibit geometric and combinatorial degeneration data which describe the geometry of the special fiber of a minimal semi-stable model of $\mathcal{Y}$.
\end{abstract}

\section{Introduction}

In this paper, which is a sequel to [13] and [14], we study the semi-stable reduction of Galois covers of degree $p$ above proper and smooth curves over a complete discrete valuation ring of mixed characteristics $(0, p)$.

More precisely, let $p>0$ be a prime integer. Let $R$ be a complete discrete valuation ring with fraction field $K$ of characteristic 0 , and residue field $k$ of characteristic $p$ which we assume to be algebraically closed. Let $\mathcal{X}$ be a proper and smooth $R$-curve with generic fiber $\mathcal{X}_{K}:=\mathcal{X} \times{ }_{R} K$ and special fiber $\mathcal{X}_{k}:=\mathcal{X} \times_{R} k$. Let $f: \mathcal{Y} \rightarrow \mathcal{X}$ be a finite Galois cover with Galois group $G$ and with $\mathcal{Y}$ normal. Let $\mathcal{Y}_{K}:=\mathcal{Y} \times_{R} K$ be the generic fiber of $\mathcal{Y}$, and let $\mathcal{Y}_{k}:=\mathcal{Y} \times_{R} k$ be its special fiber which we assume to be reduced (this condition is always satisfied after a finite extension of $R$ ). If the cardinality of $G$ is prime to $p$ and if the cover $f_{K}: \mathcal{Y}_{K} \rightarrow \mathcal{X}_{K}$ between generic fibers is étale then it follows from the purity theorem that $\mathcal{Y}$ is smooth (cf. [16]). If

Communicated by A. Tamagawa. Received December 2, 2005. Revised October 25, 2006. 2000 Mathematics Subject Classification(s): 14H30, 14H20, 11G20.

*School of Engineering, Computer Science and Mathematics, Harrison Building, North Park Road, Exeter EX44QF, U.K.

e-mail: M.Saidi@exeter.ac.uk 
the cardinality of $G$ is divisible by $p$ then $\mathcal{Y}$ is not smooth in general (even if the cover $f_{K}$ between generic fibers is étale). However, it follows from the theorem of semi-stable reduction of curves (cf. [4]) that $\mathcal{Y}$ admits potentially semi-stable reduction, i.e. there exists (possibly after extending $R$ ) a proper and birational morphism $\tilde{\mathcal{Y}} \rightarrow \mathcal{Y}$ where $\tilde{\mathcal{Y}}$ is a semi-stable $R$-curve. Moreover, there exists such a semi-stable model $\tilde{\mathcal{Y}}$ which is minimal (cf. discussion in 3.1). Our main interest is in the study of the geometry (of the special fiber) of a minimal semi-stable model $\tilde{\mathcal{Y}}$ under the assumption that $p$ divides the cardinality of $G$. The first result in this direction is the following, which is due to Raynaud (cf. [12]):

Theorem (Raynaud). $\quad$ Assume that $G$ is a p-group and that the cover $f$ is étale above the generic fiber $\mathcal{X}_{K}$ of $\mathcal{X}$. Then the configuration of the special fiber $\tilde{\mathcal{Y}}_{k}:=\tilde{\mathcal{Y}} \times_{R} k$ of a minimal semi-stable model $\tilde{\mathcal{Y}}$ of $\mathcal{Y}$ is tree-like.

More generally, this result holds under the assumption that $f$ is étale above $U_{K}:=X_{K}-D_{K}$, where $D_{K}$ is the generic fiber of a divisor $D \subset X$, which is étale over $R$. Moreover, Raynaud proves that the ends of the tree $\tilde{\mathcal{Y}}_{k}$ are étale covers of the affine line. Our aim in this paper is to complement and generalize Raynaud's result in the case where $G \simeq \mathbb{Z} / p \mathbb{Z}$. Under no assumption on the ramification locus in the morphism $f_{K}: Y_{K} \rightarrow X_{K}$, we exhibit some extra geometric and combinatorial data which describe the geometry of the minimal semi-stable model of $Y$ (compare with [3], [5], and [10] where analogous problems are treated).

This paper is organized as follows. We first start in Sections 1 and 2 by recalling the main results in [13] (resp. [14]), which concern the degeneration of $\mu_{p}$-torsors from 0 to positive characteristic (resp. the computation of vanishing cycles in a Galois cover of degree $p$ between formal germs of $R$-curves). As a consequence of these results we can determine the singular points of $\mathcal{Y}_{k}$ and we can compute the arithmetic genus of these singularities. More precisely, let $B_{k} \subset \mathcal{X}_{k}$ be the set of specialization of the branch locus in the morphism $f_{K}: \mathcal{Y}_{K} \rightarrow \mathcal{X}_{K}$ and let $U_{k}^{\prime}:=\mathcal{X}_{k}-B_{k}$. Then $f$ induces (by restriction to $\left.U_{k}^{\prime}\right)$ a finite cover $f_{k}^{\prime}: V_{k}^{\prime} \rightarrow U_{k}^{\prime}$ which has the structure of a torsor under a finite and flat $k$-group scheme of rank $p$ (cf. 1.3). Suppose for example that this torsor is radicial (this is the most difficult case to treat) and let $\omega$ be the associated differential form (cf. [13], 1). Let $Z_{k}$ be the set of zeros of $\omega$ and let $\operatorname{Crit}(f):=Z_{k} \cup B_{k}$. If $y$ is a singular point of $\mathcal{Y}_{k}$ then $f(y) \in \operatorname{Crit}(f)$. Let $m_{y}:=\operatorname{ord}_{f(y)}(\omega)$. Then the arithmetic genus of $y$ (cf. [14],3.1) equals 
$\left(r_{y}+m_{y}\right)(p-1) / 2$, where $r_{y}$ is the number of branched points of $f$ in the generic fiber $\mathcal{X}_{K}$ which specialize in $f(y)\left(r_{y}=0\right.$ if $\left.f(y) \in \operatorname{Crit}(f)-B_{k}\right)$.

Further, in order to understand the geometry of $\tilde{\mathcal{Y}}$ one needs to understand the fiber of a singular point $y$ of $\mathcal{Y}_{k}$ in the minimal semi-stable model $\tilde{\mathcal{Y}}$. This is, indeed, a local problem which we study in Section 3. There we consider a finite Galois cover $f_{x}: \mathcal{Y}_{y} \rightarrow \mathcal{X}_{x}$ of degree $p$ between formal germs of $R$-curves at a closed point $y$ (resp. $x$ ), where $x$ is a smooth point (i.e. $\mathcal{X}_{x} \simeq \operatorname{Spf} R[[T]]$ ), and we study the geometry of a minimal semi-stable model $\tilde{\mathcal{Y}}_{y}$ of $\mathcal{Y}_{y}$. In 3.2 we exhibit what we call "simple degeneration data of rank $p$ torsor", comprising a tree $\Gamma$ of $k$-projective lines which is endowed with some data of geometric and combinatorial nature and which completely describe the geometry of $\tilde{\mathcal{Y}}_{y}$. More precisely, let $\mathbf{D e g}_{\mathbf{p}}$ be the set of "isomorphism classes" of such data (cf. Definition 3.4). Then we construct a canonical specialization map $\mathrm{Sp}$ : $H_{\mathrm{et}}^{1}(\operatorname{Spec} L, \mathbb{Z} / p \mathbb{Z}) \rightarrow \operatorname{Deg}_{\mathbf{p}}$, where $L$ is the function field of $\overline{\mathcal{X}}_{x}:=\mathcal{X}_{x} \times_{R} \bar{R}$ and $\bar{R}$ is the integral closure of $R$ in an algebraic closure of $K$. Our first main result in this paper is the realization of simple degeneration data. More precisely, we prove the following.

Theorem (cf. 3.6). The specialization map $\mathrm{Sp}: H_{\mathrm{et}}^{1}(\operatorname{Spec} L, \mathbb{Z} / p \mathbb{Z}) \rightarrow$ Deg $_{\mathbf{p}}$ defined in 3.4 is surjective.

In other words, we are able to reconstruct Galois covers of degree $p$ above open $p$-adic discs starting from (the) degeneration data which describe the semi-stable reduction of such a cover. The proof of this result relies on the technique of formal patching initiated by Harbater and Raynaud (cf. [14], 1). The above theorem was proven in [5] under the assumption that $\mathcal{Y}_{y}$ is smooth, and $\tilde{\mathcal{Y}}_{y}$ is the minimal semi-stable model in which the ramified points (on the generic fiber) specialize in smooth distinct points. In 3.7, as an application of Theorem 3.6, we give an example of a Galois $p$-cover $f_{x}: \mathcal{Y}_{y} \rightarrow \mathcal{X}_{x}$ as above where the configuration of a semi-stable model of $\mathcal{Y}_{y}$ is not tree-like.

In Section 4 we return to the above global situation of a Galois cover $f: \mathcal{Y} \rightarrow \mathcal{X}$ of degree $p$. The results in Section 3 allow us to associate with each critical point $x_{i}=f\left(y_{i}\right) \in \operatorname{Crit}(f)$ simple degeneration data $\operatorname{Deg}\left(x_{i}\right)$ of rank $p$ torsor, which describe the preimage of the singular point $y_{i}$ in $\tilde{\mathcal{Y}}_{k}$. These simple degeneration data, plus the data given by the torsor $f_{k}^{\prime}: V_{k}^{\prime} \rightarrow U_{k}^{\prime}$, lead to the definition of "smooth degeneration data" $\operatorname{Deg}\left(\mathcal{X}_{k}\right)$ of rank $p$ torsor, which are associated with the special fiber $\mathcal{X}_{k}$ of $\mathcal{X}$ and which describe the geometry of the semi-stable model $\tilde{\mathcal{Y}}$ of $\mathcal{Y}$. More precisely, let $\mathbf{D E G}_{\mathbf{p}}\left(\mathcal{X}_{\mathbf{k}}\right)$ be the set of 
isomorphism classes of smooth degeneration data of rank $p$ torsor associated with $\mathcal{X}_{k}$ (cf. Definition 4.3). Then we construct a canonical "specialization" map Sp : $H_{\text {et }}^{1}(\operatorname{Spec} L, \mathbb{Z} / p \mathbb{Z}) \rightarrow \mathbf{D E G}_{\mathbf{p}}\left(\mathcal{X}_{\mathbf{k}}\right)$, where $L$ is the function field of $\overline{\mathcal{X}}:=\mathcal{X} \times_{R} \bar{R}$ and $\bar{R}$ is the integral closure of $R$ in an algebraic closure of $K$. Our second main result is the realization of smooth degeneration data associated with $\mathcal{X}_{k}$, if necessary after modifying the $R$-curve $\mathcal{X}$ into another $R$-curve $\mathcal{X}^{\prime}$ with special fiber $\mathcal{X}^{\prime}{ }_{k}$ isomorphic to $\mathcal{X}_{k}$. More precisely, we have the following.

Theorem (cf. 4.5). Let $\operatorname{Deg}\left(\mathcal{X}_{k}\right) \in \mathbf{D E G}_{\mathbf{p}}\left(\mathcal{X}_{\mathbf{k}}\right)$ be smooth degeneration data of rank $p$ torsor associated with $\mathcal{X}_{k}$. Then there exists a smooth and proper $R$-curve $\mathcal{X}^{\prime}$ with special fiber isomorphic to $\mathcal{X}_{k}$ such that $\operatorname{Deg}\left(\mathcal{X}_{k}\right)$ is in the image of the specialization map $\mathrm{Sp}: H_{\mathrm{et}}^{1}(\operatorname{Spec} L, \mathbb{Z} / p \mathbb{Z}) \rightarrow \mathbf{D E G}_{\mathbf{p}}\left(\mathcal{X}_{\mathbf{k}}\right)$, where $L$ is the function field of $\mathcal{X}^{\prime} \times_{R} \bar{R}$ and $\bar{R}$ is the integral closure of $R$ in an algebraic closure of $K$.

As another application of our techniques we prove the following result of lifting of torsors under finite and flat group schemes of rank $p$ (this result is also proved in [1] using different methods). In fact Theorem 4.5 is a stronger version of Theorem 4.7 (cf. Remark 4.8,1).

Theorem (cf. 4.7). $\quad$ Let $X$ be a smooth and proper $k$-curve and let $f$ : $Y \rightarrow X$ be a torsor under a finite and flat $k$-group scheme $G_{k}$ of rank $p$. Then there exists a smooth and proper $R$-curve $\mathcal{X}$, with special fiber isomorphic to $X$, and a torsor $\tilde{f}: \mathcal{Y} \rightarrow \mathcal{X}$ under an $R$-group scheme $G_{R}$, which is commutative finite and flat of rank $p$, such that the torsor induced on the level of special fibers $\tilde{f}_{k}: \mathcal{Y}_{k} \rightarrow \mathcal{X}_{k}$ is isomorphic to the torsor $f$. In other words the torsor $\tilde{f}$ lifts $f$.

In this paper we do not address questions of "effectiveness". Namely is it possible for a given Galois $p$-cover $f: \mathcal{Y} \rightarrow \mathcal{X}$ as above (say given by explicit equations) to determine explicitly the smooth degeneration data which describes the geometry of the minimal semi-stable model of $\mathcal{Y}$ ? What is needed for this is a recepee for getting centers for the appropriate blowing up necessary to obtain the minimal semi-stable model, and to deduce the monodromy extension i.e. the minimal extension $K^{\prime} / K$ over which an equivariant semi-stable model exists. These questions are studied in [6], [7], and [9], in the case where $\mathcal{X}$ is the $R$-projective line and under some (restrictive) conditions on the branch 
locus.

It is important to be able to extend the results of this paper to the more general case where the Galois group $G \simeq \mathbb{Z} / p^{n} \mathbb{Z}$ is cyclic of order $p^{n}$. However, what is really missing is a generalization of the results in section 1 to this case, in other words to describe the way $\mu_{p^{n}}$-torsors degenerate from characteristic 0 to characteristic $p$. Examples in the case $n=2$ already illustrate the complexity of the situation, by comparison with the case $n=1$

\section{$\S 1$. Degeneration of $\mu_{p}$-Torsors from Characteristic 0 to Characteristic $p>0$}

In this section we recall the degeneration of $\mu_{p}$-torsors from zero to positive characteristic (cf. [13], II, III, for more details) which plays an important role in later sections. We fix the following notation: $R$ is a complete discrete valuation ring of unequal characteristic with residue characteristic $p>0$, which contains a primitive $p$-th root of unity $\zeta$. We denote by $K$ the fraction field of $R, \pi$ a uniformizing parameter, $\lambda:=\zeta-1$, and by $k$ the residue field which we assume to be algebraically closed. We also denote by $v_{K}$ the valuation of $K$ which is normalized by $v_{K}(\pi)=1$.

\section{$\S 1.1$. Torsors under finite and flat $R$-group schemes of rank $p$ : the group schemes $\mathcal{G}^{n}$ and $\mathcal{H}_{n}$}

For a positive integer $n$, let $\mathcal{G}_{R}^{n}$ be the affine $R$-group scheme

$$
\operatorname{Spec} R\left[x, 1 /\left(\pi^{n} x+1\right)\right]
$$

It is a commutative affine group scheme whose generic fiber is isomorphic to the multiplicative group scheme $\mathbb{G}_{m, K}$, and whose special fiber is the additive group $\mathbb{G}_{a, k}$ (cf. [11] for more details). For $0<n \leq v_{K}(\lambda)$ the polynomial

$$
\left(\left(\pi^{n} x+1\right)^{p}-1\right) / \pi^{p n}
$$

has coefficients in $R$ and defines a group scheme homomorphism

$$
\phi_{n}: \mathcal{G}_{R}^{n} \rightarrow \mathcal{G}_{R}^{p n}
$$

which at the level of corresponding Hopf algebras is given by

$$
\begin{gathered}
\operatorname{Spec} R\left[x, 1 /\left(\pi^{n p} x+1\right)\right] \rightarrow \operatorname{Spec} R\left[x, 1 /\left(\pi^{n} x+1\right)\right] \\
x \rightarrow\left(\left(\pi^{n} x+1\right)^{p}-1\right) / \pi^{p n}
\end{gathered}
$$


The homomorphism $\phi_{n}$ is an isogeny of degree $p$. Its kernel $\mathcal{H}_{n, R}$ is a finite and flat $R$-group scheme of rank $p$ whose generic fiber is isomorphic to $\mu_{p}$ and whose special fiber is either the radicial group scheme $\alpha_{p}$ if $0<n<v_{K}(\lambda)$, or the étale group scheme $\mathbb{Z} / p \mathbb{Z}$ if $n=v_{K}(\lambda)$. More precisely, the exact sequence

$$
0 \longrightarrow \mathcal{H}_{n, R} \longrightarrow \mathcal{G}_{R}^{n} \stackrel{\phi_{n}}{\longrightarrow} \mathcal{G}_{R}^{n p} \longrightarrow 0
$$

induces on the generic fiber an exact sequence which is isomorphic to the Kummer sequence

$$
0 \longrightarrow \mu_{p, K} \longrightarrow \mathbb{G}_{m, K} \stackrel{x^{p}}{\longrightarrow} \mathbb{G}_{m, K} \longrightarrow 0
$$

and induces on the special fiber the exact sequence

$$
0 \longrightarrow \alpha_{p, k} \longrightarrow \mathbb{G}_{a, k} \stackrel{x^{p}}{\longrightarrow} \mathbb{G}_{a, k} \longrightarrow 0
$$

if $n<v_{K}(\lambda)$, and the Artin-Schreier sequence

$$
0 \longrightarrow \mathbb{Z} / p \mathbb{Z} \longrightarrow \mathbb{G}_{a, k} \stackrel{x^{p}-x}{\longrightarrow} \mathbb{G}_{a, k} \longrightarrow 0
$$

if $n=v_{K}(\lambda)$.

Let $\psi^{(n)}: \mathcal{G}_{R}^{n} \rightarrow \mathbb{G}_{m, R}$ be the group scheme homomorphism given on the corresponding Hopf algebras by

$$
\begin{aligned}
R\left[u, u^{-1}\right] & \rightarrow R\left[x, 1 /\left(\pi^{n} x+1\right)\right] \\
u & \rightarrow \pi^{n} x+1
\end{aligned}
$$

The following is a commutative diagram of exact sequences for the fppftopology.

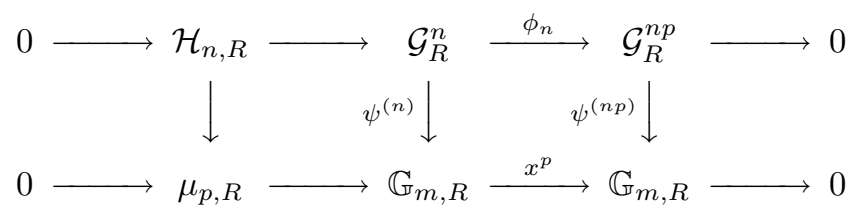

The above diagram leads to the following description for $\mathcal{H}_{n, R}$-torsors in the fppf-topology. Let $\mathcal{U}$ be an $R$-scheme (or a formal $R$-scheme) and let $f: \mathcal{V} \rightarrow \mathcal{U}$ be a torsor under the group scheme $\mathcal{H}_{n, R}$. Then there exists an open covering $\left(\mathcal{U}_{i}\right)$ of $\mathcal{U}$ and regular functions $u_{i} \in \Gamma\left(\mathcal{U}_{i}, \mathcal{O}_{\mathcal{U}}\right)$, where $\pi^{n p} u_{i}+1$ is defined up to multiplication by a $p$-power, such that the torsor $f$ is defined above $\mathcal{U}_{i}$ by the equation

$$
T_{i}^{p}=\left(\pi^{n} T_{i}^{\prime}+1\right)^{p}=\pi^{n p} u_{i}+1
$$


where $T_{i}$ and $T_{i}^{\prime}$ are indeterminates. In particular, the torsor $f_{k}: \mathcal{V}_{k} \rightarrow \mathcal{U}_{k}$ at the level of special fibers is locally given by the equation

$$
t_{i}^{\prime p}=\bar{u}_{i}
$$

if $n<v_{K}(\lambda)$, resp.

$$
t_{i}^{\prime p}-t_{i}^{\prime}=\bar{u}_{i}
$$

if $n=v_{K}(\lambda)$, where $\bar{u}_{i}$ is the image of $u_{i}$ modulo $\pi$.

\section{$\S 1.2$. Degeneration of $\mu_{p}$-torsors}

In what follows let $X$ be a formal $R$-scheme of finite type which is normal, flat, and connected over $R$. Assume that $X$ is smooth of relative dimension 1 . Let $X_{K}:=X \times_{R} K$ (resp. $X_{k}:=X \times_{R} k$ ) be the generic (resp. the special) fiber of $X$. By the generic fiber of $X$ we mean the associated $K$-rigid space (cf. $[2])$. Let $\eta$ be the generic point of the special fiber $X_{k}$. The localization $\mathcal{O}_{X, \eta}$ of $X$ at $\eta$ is a discrete valuation ring with fraction field $K(X)$ the function field of $X$. The following result describes the way in which $\mu_{p}$-torsors, above the generic fiber $X_{K}$ of $X$, degenerate.

Proposition 1.3. Let $f_{K}: Y_{K} \rightarrow X_{K}$ be a $\mu_{p}$-torsor with $Y_{K}$ connected and let $K(X) \rightarrow F$ be the corresponding extension of function fields. Assume that the ramification index above $\mathcal{O}_{X, \eta}$ in the extension $K(X) \rightarrow F$ equals 1 . Then the torsor $f_{K}: Y_{K} \rightarrow X_{K}$ extends to a torsor $f: Y \rightarrow X$ under a finite and flat $R$-group scheme of rank $p$, with $Y$ normal. Let $\delta$ be the degree of the different above $\eta$ in the extension $K(X) \rightarrow F$. Then the following cases occur. a) $\delta=0$. In this case $f$ is a torsor under the group scheme $\mathcal{H}_{v_{K}(\lambda), R}$ and $f_{k}: Y_{k} \rightarrow X_{k}$ is an étale torsor under $\mathbb{Z} / p \mathbb{Z}$.

b) $0<\delta<v_{K}(\lambda)$. In this case $\delta=v_{K}(\lambda)-n(p-1)$, for a certain integer $n \geq 1, f$ is a torsor under the group scheme $\mathcal{H}_{n, R}$ and $f_{k}$ is a radicial torsor under $\alpha_{p}$.

c) $\delta=v_{K}(\lambda)$. In this case $f$ is a torsor under $\mu_{p}$ and $f_{k}$ is also a torsor under $\mu_{p}$.

Proof. Cf. [13], 2.4.

Remark 1.4. The above result is false in general. More precisely, for

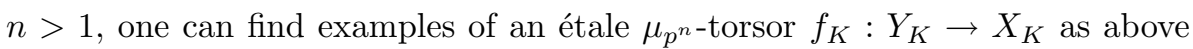
which doesn't extend to a torsor $f: Y \rightarrow X$ under a finite and flat $R$-group scheme of rank $p^{n}$ (and even not on any finite extension of $R$ ). In [15] we 
produced such examples in equal characteristic $p>0$, for $n=2$. However, this result remains always true for abelian torsors (i.e. torsors under a commutative group scheme) if $X$ is proper, as is easily seen by using the relative Picard varieties. I do not know whether it remains true for non-abelian torsors in the proper case.

\section{$\S 1.5$. Degeneration of $\mu_{p}$-torsors on the boundaries of formal fibres}

In what follows we recall the degeneration of $\mu_{p}$-torsors on a boundary

$$
\mathcal{X} \simeq \operatorname{Spf} R[[T]]\left\{T^{-1}\right\}
$$

of germs of formal $R$-curves (cf. [14], 1 and 2 for more details). Here, $R[[T]]$ $\left\{T^{-1}\right\}$ denotes the ring of formal power series $\sum_{i \in \mathbb{Z}} a_{i} T^{i}$ with $\lim _{i \rightarrow-\infty} v_{K}\left(a_{i}\right)=$ $\infty$. The element $T$ of $A:=R[[T]]\left\{T^{-1}\right\}$ is called a parameter of $A$. Note that $R[[T]]\left\{T^{-1}\right\}$ is a complete discrete valuation ring with uniformizing parameter $\pi$ and residue field $k((t))$, where $t \equiv T \bmod \pi$. The following result describes the way $\mu_{p}$-torsors above the generic fiber of a boundary $\mathcal{X} \simeq \operatorname{Spf} R[[T]]\left\{T^{-1}\right\}$ of a formal fiber (or germ) degenerate. This result plays an essential role in the computation of vanishing cycles in a Galois cover of degree $p$ between formal fibers.

Proposition 1.6. Let $A:=R[[T]]\left\{T^{-1}\right\}$ (cf. the above definition) and let

$$
f: \operatorname{Spf} B \rightarrow \operatorname{Spf} A
$$

be a (non-trivial) Galois cover of degree $p$. Assume that the ramification index of the corresponding extension of discrete valuation rings equals 1 . Then $f$ is a torsor under a finite and flat $R$-group scheme $G$ of rank $p$. Let $\delta$ be the degree of the different in the above extension. The following cases occur.

a) $\delta=v_{K}(p)$. In this case $f$ is a torsor under $G=\mu_{p}$ and two cases can occur:

a-1) For a suitable choice of the parameter $T$ of $A$ the torsor $f$ is given, if necessary after a finite extension of $R$, by an equation $Z^{p}=T^{h}$.

a-2) For a suitable choice of the parameter $T$ of $A$ the torsor $f$ is given, if necessary after a finite extension of $R$, by an equation $Z^{p}=1+T^{m}$, where $m$ is a positive integer prime to $p$.

b) $0<\delta<v_{K}(p)$. In this case $f$ is a torsor under the group scheme $G=\mathcal{H}_{n, R}$, where $n$ is such that $\delta=v_{K}(p)-n(p-1)$. Moreover, for a suitable choice of the parameter $T$, the torsor $f$ is given, if necessary after a finite extension of $R$, by an equation $Z^{p}=1+\pi^{n p} T^{m}$ where $m \in \mathbb{Z}$ is prime to $p$. 
c) $\delta=0$. In this case $f$ is an étale torsor under the $R$-group scheme $G=\mathcal{H}_{v_{K}(\lambda), R}$ and is given, if necessary after a finite extension of $R$, by an equation $Z^{p}=1+\lambda^{p} T^{m}$, where $m$ is a negative integer prime to $p$ (for a suitable choice of the parameter $T$ of $A$ ).

Proof. Cf. [14], 2.3.

Definition 1.7 (cf. also [14] 2.4). With the same notation as in 1.6 we define the reduction type (or the degeneration type) of the torsor $f$ to be $\left(G_{k},-m, h\right)$. Here $G_{k}:=G \times_{R} k$ is the special fiber of the group scheme $G$, $m$ is the "conductor" associated with the torsor $f_{k}: \operatorname{Spec} B / \pi B \rightarrow \operatorname{Spec} A / \pi A$ which appears in 1.6 (setting $m=0$ in the case a-1)), and $h \in \mathbb{F}_{p}$ is its "residue" which equals 0 apart from the case a-1) where it equals the residue of the differential form $d\left(t^{h}\right) / t^{h}$. Following the notation of 1.6 the degeneration type is then $\left(\mu_{p}, 0, h\right)$ in case a- 1$),\left(\mu_{p},-m, 0\right)$ in case a-2), $\left(\alpha_{p},-m, 0\right)$ in case b), and $(\mathbb{Z} / p \mathbb{Z},-m, 0)$ in case $\mathrm{c})$.

\section{$\S 2 . \quad$ Computation of Vanishing Cycles}

In this section we recall the results established in [14] concerning the computation of vanishing cycles in a Galois cover of degree $p$ between formal germs of formal $R$-curves. These results play a key role in establishing the main theorems of this paper. We only recall the results concerning covers above formal germs of a semi-stable curve and refer to [14], 3, for the general case. We start with the case of a smooth point.

Proposition 2.1. Let $\mathcal{X}:=\operatorname{Spf} R[[T]]$ be the germ of a formal $R$-curve at a smooth point $x$ and let $\mathcal{X}_{\eta}:=\operatorname{Spf} R[[T]]\left\{T^{-1}\right\}$ be the boundary of $\mathcal{X}$. Let $f: \mathcal{Y} \rightarrow \mathcal{X}$ be a Galois cover of degree $p$ with $\mathcal{Y}$ local. Assume that the special fiber of $\mathcal{Y}$ is reduced and let $y$ be the unique closed point of $\mathcal{Y}_{k}$. We denote by $g_{y}$ the genus of $y$ ( $c f$. [14], 3.1 for the definition of $\left.g_{y}\right)$. Let $\delta_{K}:=r(p-1)$ be the degree of the divisor of ramification in the morphism $f: \mathcal{Y}_{K} \rightarrow \mathcal{X}_{K}$. We distinguish two cases.

case 1) $\mathcal{Y}_{k}$ is unibranch at $y$. Let $\left(G_{k}, m, h\right)$ be the degeneration type of $f$ above the boundary $\mathcal{X}_{\eta}$ (cf. 1.7). Then necessarily $r-m-1 \geq 0$ and $g_{y}=(r-m-1)(p-1) / 2$.

case 2) $\mathcal{Y}_{k}$ has $p$-branches at $y$. Then the cover $f$ has an étale split reduction on the boundary, i.e. the induced torsor above $\operatorname{Spf} R[[T]]\left\{T^{-1}\right\}$ is trivial, in which case $g_{y}=(r-2)(p-1) / 2$. 
As an immediate consequence of 2.1 one can determine whether the point $y$ is smooth or not. More precisely, we have the following.

Corollary 2.2. We use the same notation as in 2.1. Then y is a smooth point, which is equivalent to $g_{y}=0$, if and only if $r=m+1$. In particular, if the reduction is of multiplicative type on the boundary, i.e. if $G_{k}=\mu_{p}$, then $g_{y}=0$ only if $r=1$ or $r=0$, since $m$ is positive in this case. Also, if $r=1$ and $g_{y}=0$ then necessarily $G_{k}=\mu_{p}$.

Next we consider the case of an ordinary double point.

Proposition 2.3. Let $\mathcal{X}:=\operatorname{Spf} R[[S, T]] /\left(S T-\pi^{e}\right)$ be the formal germ of an $R$-curve at an ordinary double point $x$ of thickness $e$ and let $\mathcal{X}_{1}:=$ Spf $R[[S]]\left\{S^{-1}\right\}, \mathcal{X}_{2}:=\operatorname{Spf} R[[T]]\left\{T^{-1}\right\}$, be the boundaries of $\mathcal{X}$. Let $f$ : $\mathcal{Y} \rightarrow \mathcal{X}$ be a Galois cover with group $\mathbb{Z} / p \mathbb{Z}$ and with $\mathcal{Y}$ local. Assume that the special fiber of $\mathcal{Y}$ is reduced and that $\mathcal{Y}_{k}$ has two branches at the point $y$. Let $\delta_{K}:=r(p-1)$ be the degree of the divisor of ramification in the morphism $f: Y_{K} \rightarrow X_{K}$. Let $\left(G_{k, i}, m_{i}, h_{i}\right)$ be the degeneration type on the boundaries of $\mathcal{X}$ for $i \in\{1,2\}$. Then necessarily $r-m_{1}-m_{2} \geq 0$ and $g_{y}=\left(r-m_{1}-m_{2}\right)(p-1) / 2$.

With the same notation as in 2.3 one can recognize whether the point $y$ is a double point or not. More precisely, we have the following.

Corollary 2.4. We use the same notation as in 2.3. Then $y$ is an ordinary double point, which is equivalent to $g_{y}=0$, if and only if $x$ is an ordinary double point of thickness divisible by $p$ and $r=m_{1}+m_{2}$. Moreover, if $g_{y}=0, r=0$, and if $\left(G_{k, i}, m_{i}, h_{i}\right)$ is the reduction type on the boundary for $i \in\{1,2\}$, then necessarily $h_{1}+h_{2}=0$.

\section{$\S 3 . \quad$ Semi-Stable Reduction of Cyclic $p$-Covers above Formal Germs of Curves in Mixed Characteristic}

In this section we use the same notation as in Section 2. Moreover, for an $R$-scheme (resp. a formal $R$-scheme) $\mathcal{X}$ we denote by $\mathcal{X}_{k}:=\mathcal{X} \times{ }_{R} k$ (resp. $\mathcal{X}_{K}:=\mathcal{X} \times{ }_{R} K$ ) the special fiber of $\mathcal{X}$ (resp. its generic fiber, which in the case where $\mathcal{X}$ is formal means the associated rigid space (cf. [2]))

3.1. Let $\mathcal{X}$ be either a formal semi-stable $R$-curve or the formal germ of a semi-stable $R$-curve $X$ at a closed point $x$, and let $f: \mathcal{Y} \rightarrow \mathcal{X}$ be a Galois cover, with group $G$, such that $\mathcal{Y}$ is normal (If $\mathcal{X}$ is a germ we also require $\mathcal{Y}$ to be 
local). In this paper we are mainly concerned with the case where $G$ is cyclic of order $p$. Then it follows easily from the theorem of semi-stable reduction for curves (cf. [4]) (as well as from the compactification process, established in [14], 3.3 , in the case of a germ) that after perhaps a finite extension $R^{\prime}$ of $R$, with fraction field $K^{\prime}$, the formal curve (resp. germ) $\mathcal{Y}$ has semi-stable reduction over $K^{\prime}$. More precisely, there exists a birational and proper morphism $\tilde{f}: \tilde{\mathcal{Y}} \rightarrow \mathcal{Y}^{\prime}$, where $\mathcal{Y}^{\prime}$ is the normalization of $\mathcal{Y} \times{ }_{R} R^{\prime}$, such that $\tilde{\mathcal{Y}}_{K^{\prime}} \simeq \mathcal{Y}_{K^{\prime}}^{\prime}$ and the following conditions hold.

(i) The special fiber $\tilde{\mathcal{Y}}_{k}:=\tilde{\mathcal{Y}} \times_{\operatorname{Spec} R^{\prime}}$ Spec $k$ of $\tilde{\mathcal{Y}}$ is reduced.

(ii) $\tilde{\mathcal{Y}}_{k}$ has only ordinary double points as singularities.

Moreover, there exists such a semi-stable model $\tilde{f}: \tilde{\mathcal{Y}} \rightarrow \mathcal{Y}^{\prime}$ which is minimal for the above properties (cf. [12], p. 182, and [8] Theorem 2.3 and Corollary 2.5). In particular, the action of $G$ on $\mathcal{Y}^{\prime}$ extends to an action on $\tilde{\mathcal{Y}}$. Let $\tilde{\mathcal{X}}$ be the quotient of $\tilde{\mathcal{Y}}$ by $G$, which is a semi-stable model of $\mathcal{X}^{\prime}:=\mathcal{X} \times_{R} R^{\prime}$. One has the following commutative diagram:

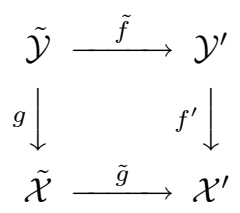

where $f^{\prime}$ is naturally induced by $f$. One can also choose the semi-stable models $\tilde{\mathcal{Y}}$ and $\tilde{\mathcal{X}}$ above so that the set of points $B_{K^{\prime}}:=\left\{x_{i, K^{\prime}}\right\}_{1 \leq i \leq r}$ consisting of the branch locus in the morphism $f_{K}^{\prime}: \mathcal{Y}_{K^{\prime}}^{\prime} \rightarrow \mathcal{X}_{K^{\prime}}^{\prime}$ specialize in smooth distincts points of $\mathcal{X}_{k}^{\prime}$. Further, we assume that $B_{K^{\prime}} \subset \mathcal{X}_{K^{\prime}}^{\prime}\left(K^{\prime}\right)$. Moreover, one can choose such $\tilde{\mathcal{X}}$ and $\tilde{\mathcal{Y}}$ which are minimal for these properties (cf. loc. cit.). Further, there exists a minimal extension $K^{\prime}$ as above such that these conditions are satisfied. In what follows we always assume that $K^{\prime}, \tilde{\mathcal{X}}$, and $\tilde{\mathcal{Y}}$ satisfy these later properties and are minimal in the above sense.

In the case where $\mathcal{X}$ is the germ of a formal semi-stable $R$-curve $X$, at a closed point $x$, the fiber $\tilde{g}^{-1}(x)$ of the closed point $x$ in $\tilde{\mathcal{X}}$ is a tree $\Gamma$ of projective lines. This tree is canonically endowed with some "degeneration data" that we will exhibit below and in the next section, in the case where $G \simeq \mathbb{Z} / p \mathbb{Z}$, and which take into account the geometry of the special fiber $\tilde{\mathcal{Y}}_{k}$ of $\tilde{\mathcal{Y}}$. The existence of these data will follow mainly from the results which we recalled in Sections 1 and 2 .

3.2. We use the notation in 3.1. We consider the case where $\mathcal{X} \simeq \operatorname{Spf} A$ is the formal germ of a semi-stable $R$-curve $X$ at a smooth point $x$, i.e. $A$ is (non-canonically) isomorphic to $R[[T]]$. Let $R^{\prime}$ be a finite extension of $R$ as 
in 3.1 and let $\pi^{\prime}$ be a uniformizer of $R^{\prime}$. Below we exhibit the degeneration data associated with the semi-stable reduction $\tilde{\mathcal{Y}}$ of $\mathcal{Y}$.

Deg.1. Let $\wp:=\left(\pi^{\prime}\right)$ be the ideal of $A^{\prime}:=A \otimes_{R} R^{\prime}$ generated by $\pi^{\prime}$ and let $\hat{A}_{\wp}^{\prime}$ be the completion of the localization of $A^{\prime}$ at $\wp$. Let $\mathcal{X}_{\eta}^{\prime}:=\operatorname{Spf} \hat{A}_{\wp}^{\prime}$ be the formal boundary of $\mathcal{X}^{\prime}$ and let $\mathcal{X}_{\eta}^{\prime} \rightarrow \mathcal{X}^{\prime}$ be the canonical morphism. Consider the following cartesian diagram:

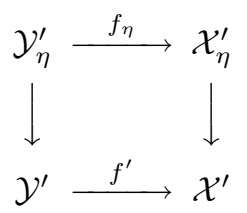

Then $f_{\eta}: \mathcal{Y}_{\eta}^{\prime} \rightarrow \mathcal{X}_{\eta}^{\prime}$ is a torsor under a finite commutative and flat $R^{\prime}$-group scheme $G_{R^{\prime}}$ of rank $p$, which is either isomorphic to $\mu_{p}$ or isomorphic to the group scheme $\mathcal{H}_{n, R^{\prime}}$ (cf. 1.1) for some integer $0<n \leq v_{K^{\prime}}(\lambda)$, as follows from 1.3. Let $\left(G_{k}, m, h\right)$ be the degeneration type of the torsor $f_{\eta}$ (cf. 1.7) which is canonically associated with $f$. Note that $G_{k}$ is isomorphic to the special fiber of $G_{R^{\prime}}$. The arithmetic genus $g_{y}$ of the point $y$ equals $(r-m-1)(p-1) / 2$ (cf. 2.1), where $d_{\eta}:=r(p-1)$ is the degree of the divisor of ramification in the morphism $f_{K^{\prime}}^{\prime}: \mathcal{Y}_{K^{\prime}}^{\prime} \rightarrow \mathcal{X}_{K^{\prime}}^{\prime}$.

Deg.2. The fiber $\tilde{g}^{-1}(x)$ of the closed point $x$ of $\mathcal{X}^{\prime}$ in $\tilde{\mathcal{X}}$ is a tree $\Gamma$ of projective lines. Let $\operatorname{Vert}(\Gamma):=\left\{X_{i}\right\}_{i \in I}$ be the set of irreducible components of $\tilde{g}^{-1}(x)$, which are the vertices of the tree $\Gamma$. The tree $\Gamma$ is canonically endowed with an origin vertex $X_{i_{0}}$, which is the unique irreducible component of $\tilde{g}^{-1}(x)$ which meets the point $x$. We fix an orientation of the tree $\Gamma$ starting from $X_{i_{0}}$ in the direction of the ends.

Deg.3. For each $i \in I$, let $\left\{x_{i, j}\right\}_{j \in S_{i}}$ be the set of points of $X_{i}$ in which some points of $B_{K^{\prime}}$ specialize ( $S_{i}$ may be empty). Also let $\left\{z_{i, j}\right\}_{j \in D_{i}}$ be the set of double points of $\tilde{\mathcal{X}}_{k}$ supported by $X_{i}$. In particular, $x_{i_{0}, j_{0}}:=x$ is a double point of $\tilde{\mathcal{X}}_{k}$. We denote by $B_{k}$ the set of all points $\cup_{i \in I}\left\{x_{i, j}\right\}_{j \in S_{i}}$, which is the set of specialization of the branch locus $B_{K^{\prime}}$, and by $D_{k}$ the set of double points of $\tilde{\mathcal{X}}_{k}$. Note that by hypothesis $B_{k}$ has the same cardinality as $B_{K^{\prime}}$.

Deg.4. Let $\mathcal{U}$ be the formal sub-scheme of $\tilde{\mathcal{X}}$ obtained by removing the formal fibers of the points in $\left\{B_{k} \cup D_{k}\right\}$. Let $\left\{\mathcal{U}_{i}\right\}_{i \in I}$ be the set of connected components of $\mathcal{U}$. The restriction $g_{i}: \mathcal{V}_{i} \rightarrow \mathcal{U}_{i}$ of $g$ to $\mathcal{U}_{i}$ is a torsor under a commutative finite and flat $R^{\prime}$-group scheme $G_{i, R^{\prime}}$ of rank $p$, which is either isomorphic to $\mu_{p}$, or isomorphic to the group scheme $\mathcal{H}_{n_{i}, R^{\prime}}$ (cf. 1.1) for some integer $0<n_{i} \leq v_{K^{\prime}}(\lambda)$, as follows from 1.3. Further, $g_{i, k}: \mathcal{V}_{i, k}:=\mathcal{V}_{i} \times_{R^{\prime}} k \rightarrow$ $\mathcal{U}_{i, k}:=\mathcal{U}_{i} \times_{R^{\prime}} k$ is a torsor under the $k$-group scheme $G_{i, R^{\prime}} \times_{R^{\prime}} k$, which is 
either étale isomorphic to $(\mathbb{Z} / p \mathbb{Z})_{k}$, or radicial isomorphic to $\left(\alpha_{p}\right)_{k}$ or $\left(\mu_{p}\right)_{k}$. Moreover, when we move in the graph $\Gamma$ from a fixed vertex $X_{i}$ in the direction of a vertex $X_{i^{\prime}}$ such that $G_{i, R^{\prime}} \simeq \mu_{p}$ (following the above fixed orientation), then the corresponding integers $n_{i}$ decrease strictly (as follows from [14], 4.3.1). Note that if $S_{i}$ is non-empty then necessarily $G_{i, k} \simeq \mu_{p}$ (cf. [14], Corollary 4.1.2).

Deg.5. Each smooth point $x_{i, j} \in B_{k}$ is endowed via $g$ with a degeneration data on the boundary of the formal fiber of $\tilde{\mathcal{X}}$ at $x_{i, j}$ (in the same way that we exhibited the data in Deg.1 above). More precisely, for each point $x_{i, j}$ we have the reduction type $\left(G_{i, k}, m_{i, j}, h_{i, j}\right)$ on the boundary of the formal fiber $\tilde{\mathcal{X}}_{i, j} \simeq \operatorname{Spf} R\left[\left[T_{i, j}\right]\right]$ of $\tilde{\mathcal{X}}$ at this point which is induced by $g$. Note that $G_{i, k}$ is necessarily isomorphic to the special fiber of the group scheme $G_{i, R^{\prime}}$. Then $r_{i, j}=m_{i, j}+1$, where $r_{i, j}(p-1)$ is the contribution to $d_{\eta}$ of the points which specialize into $x_{i, j}$, as follows from 2.2 (since the point of $\tilde{\mathcal{Y}}$ above $x_{i, j}$ is smooth). In particular, $m_{i, j}=0$, since $r_{i, j}=1$, and $\sum_{j \in S_{i}} h_{i, j}=0$ (as follows from the residue theorem).

Deg.6. Each double point $z_{i, j}=z_{i^{\prime}, j^{\prime}} \in X_{i} \cap X_{i^{\prime}}$ of $\tilde{\mathcal{X}}$, with origin vertex $X_{i}$ and terminal vertex $X_{i^{\prime}}$, is endowed with degeneration data $\left(G_{i, k}, m_{i, j}, h_{i, j}\right)$ and $\left(G_{i^{\prime}, k}, m_{i^{\prime}, j^{\prime}}, h_{i^{\prime}, j^{\prime}}\right)$ induced by $g$ on the two boundaries of the formal fiber of $\tilde{\mathcal{X}}$ at this point. Also, we have $m_{i, j}+m_{i^{\prime}, j^{\prime}}=0$ as follows from 2.4 (since $r=0$ in this case, and the point of $\tilde{\mathcal{Y}}$ above $z_{i, j}$ is a double point). Let $e_{i, j}$ be the thickness of the double point $z_{i, j}$. Then $e_{i, j}=p t_{i, j}$ is necessarily divisible by $p$ and we have $n_{i}-n_{i^{\prime}}=t_{i, j} m_{i, j}$ (as follows from [14], 4.3.1).

Deg.7. It follows after easy calculation that

$$
g_{y}=\sum_{i \in I_{\mathrm{et}}}\left(-2+\sum_{j \in S_{i}}\left(m_{i, j}+1\right)+\sum_{j \in D_{i}}\left(m_{i, j}+1\right)\right)(p-1) / 2
$$

where $I_{\text {et }}$ is the subset of $I$ consisting of those $i$ for which the torsor $f_{i}$ is étale (i.e. such that $\left.G_{i, R^{\prime}} \simeq \mathcal{H}_{v_{K}(\lambda), R^{\prime}}\right)$.

Note that in case $f$ is the trivial (étale $\mathbb{Z} / p \mathbb{Z}$ )-torsor then the above degeneration data consist of a tree $\Gamma$ with only one vertex $X_{i}$, and a marked smooth point $x_{i}$, endowed with the trivial (étale $\mathbb{Z} / p \mathbb{Z}$ )-torsor above $U_{i}:=X_{i}-\left\{x_{i}\right\}$.

Remark/Example 3.3. We could also have considered the minimal semi-stable models $\tilde{\mathcal{Y}}$ and $\tilde{\mathcal{X}}$ where we assume that the branched points on the generic fiber $\tilde{\mathcal{X}} \times{ }_{R^{\prime}} K^{\prime}$ specialize into smooth (not necessarily distinct) points of $\tilde{\mathcal{X}} \times{ }_{R^{\prime}} k$ and exhibit the corresponding degeneration data in this case. In what follows we give an example of a Galois cover $f: \mathcal{Y} \rightarrow \mathcal{X}$ of degree $p$, 
where $\mathcal{X} \simeq \operatorname{Spf} R[[T]]$ is the formal germ of a smooth point and where one can exhibit these degeneration data. More precisely, for $m>0$ an integer prime to $p$ and a positive integer $n<v_{K}(\lambda)$ consider the cover $f$ given generically by the equation

$$
X^{p}=1+\lambda^{p}\left(T^{-m}+\pi T^{-m-1}\right)
$$

Here $r=m+2$, and this cover has a reduction of type $(\mathbb{Z} / p \mathbb{Z}, m, 0)$ on the boundary. In particular, the arithmetic genus $g_{y}$ of the closed point $y$ of $\mathcal{Y}$ equals $(p-1) / 2$ (cf. [14], Examples 4.1.4, (1)). The degeneration data associated with the above cover consists necessarily of a tree $\Gamma$, with only one vertex and no edges. This is thus a unique projective line $X_{1}$ with a marked point $x_{1}$ and endowed with an étale torsor (via Deg.4) $f_{1}: V_{1} \rightarrow U_{1}:=X_{1}-\left\{x_{1}\right\}$ above $U_{1}$ with conductor 2 at the point $x_{1}$.

The above considerations lead naturally to the following abstract geometric and combinatorial definition of degeneration data.

Definition 3.4. $\quad K^{\prime}$-simple degeneration data $\operatorname{Deg}(\mathrm{x})$ of type $(r,(n, m))$ and rank $p$ torsor, where $K^{\prime}$ is a finite extension of $K$, consist of the following.

Deg.1. $r \geq 0$ is an integer, $m$ is an integer prime to $p$ such that $r-m-1 \geq$ 0 , and $0 \leq n \leq v_{K^{\prime}}(\lambda)$ is an integer. Further, $G_{k}$ is a commutative finite and flat $k$-group scheme of rank $p$ which is either étale if $n=v_{K^{\prime}}(\lambda)$, radicial of type $\alpha_{p}$ if $0<n<v_{K^{\prime}}(\lambda)$, or radicial of type $\mu_{p}$ if $n=0$.

Deg.2. $\Gamma:=X_{k}$ is an oriented tree of $k$-projective lines with vertices $\operatorname{Vert}(\Gamma):=\left\{X_{i}\right\}_{i \in I}$, which is endowed with an origin vertex $X_{i_{0}}$ and a marked point $x:=x_{i_{0}, j_{0}}$ on $X_{i_{0}}$. We denote by $\left\{z_{i, j}\right\}_{j \in D_{i}}$ the set of double points, or (non oriented) edges of $\Gamma$, which are supported by $X_{i}$. Further, we assume that the orientation of $\Gamma$ is in the direction going from $X_{i_{0}}$ towards its ends.

Deg.3. For each vertex $X_{i}$ of $\Gamma$ there is a set (which may be empty) of smooth marked points $\left\{x_{i, j}\right\}_{j \in S_{i}}$.

Deg.4. For each $i \in I$, there is a torsor $f_{i}: V_{i} \rightarrow U_{i}:=X_{i}-\left\{\left\{x_{i, j}\right\}_{j \in S_{i}} \cup\right.$ $\left.\left\{z_{i, j}\right\}_{j \in D_{i}}\right\}$ under a finite commutative and flat $k$-group scheme $G_{i, k}$ of rank $p$, which is either étale or radicial of type $\alpha_{p}$ or $\mu_{p}$, with $V_{i}$ smooth. Moreover, for each $i \in I$ there is an integer $0 \leq n_{i} \leq v_{K^{\prime}}(\lambda)$ which equals $v_{K^{\prime}}(\lambda)$ if and only if $f_{i}$ is étale, and equals 0 if and only if $G_{i, k} \simeq \mu_{p}$. If $S_{i}$ is non empty we assume that $G_{i, k} \simeq \mu_{p}$.

Deg.5. For each $i \in I$, and $j \in S_{i}$, there is a pair of integers $\left(m_{i, j}, h_{i, j}\right)$, where $m_{i, j}$ (resp. $h_{i, j}$ ) is the conductor (resp. the residue) of the torsor $f_{i}$ at 
the point $x_{i, j}$ (cf. [13], I). Further, we assume that $m_{i_{0}, j_{0}}=-m, m_{i, j}=0$ if $(i, j) \neq\left(i_{0}, j_{0}\right)$, and $\sum_{j \in S_{i}} h_{i, j}=0$.

Deg.6. For each double point $z_{i, j}=z_{i^{\prime}, j^{\prime}} \in X_{i} \cap X_{i^{\prime}}$ there is an integer $m_{i, j}$ (resp. $m_{i^{\prime}, j^{\prime}}$ ) prime to $p$, where $m_{i, j}$ (resp. $m_{i^{\prime}, j^{\prime}}$ ) is the conductor of the torsor $f_{i}\left(\operatorname{resp} . f_{i^{\prime}}\right)$ at the point $z_{i, j}\left(\operatorname{resp} . z_{i^{\prime}, j^{\prime}}\right)(\mathrm{cf}$. [13], 1.3 and 1.5). These data must satisfy $m_{i, j}+m_{i^{\prime}, j^{\prime}}=0$.

Deg.7. For each double point $z_{i, j}=z_{i^{\prime}, j^{\prime}} \in X_{i} \cap X_{i^{\prime}}$ of $\Gamma$ with origin vertex $X_{i}$, there is an integer $e_{i, j}=p t_{i, j}$ divisible by $p$ such that with the same notation as above we have $n_{i}-n_{i^{\prime}}=m_{i, j} t_{i, j}$. Moreover, associated with $x$ is an integer $e=p t$ such that $n-n_{i_{0}}=m t$.

Deg.8. Let $I_{\text {et }}$ be the subset of $I$ consisting of those $i$ for which $G_{i, k}$ is étale. Then the following equality should hold: $(r-m-1)(p-1) / 2=\sum_{i \in I_{\mathrm{et}}}(-2+$ $\left.\sum_{j \in S_{i}}\left(m_{i, j}+1\right)+\sum_{j \in D_{i}}\left(m_{i, j}+1\right)\right)(p-1) / 2$. The integer $g:=(r-m-1)(p-1) / 2$ is called the genus of the degeneration data $\operatorname{Deg}(\mathrm{x})$.

Note that if $K^{\prime \prime}$ is a finite extension of $K^{\prime}$ then $K^{\prime}$-simple degeneration data $\operatorname{Deg}(x)$ can be naturally considered as $K^{\prime \prime}$-degeneration data by multiplying all integers $n, n_{i}$, and $e_{i, j}$, by the ramification index of $K^{\prime \prime}$ over $K^{\prime}$.

There is a natural notion of isomorphism of simple degeneration data of a given type and rank $p$ torsor relative to some finite extension $K^{\prime}$ of $K$. We will denote by $\operatorname{Deg}_{\mathbf{p}}$ the set of isomorphism classes of $K^{\prime}$-simple degeneration data of rank $p$ torsor, where $K^{\prime}$ runs over all finite extensions $K^{\prime}$ of $K$. The above discussion in 3.2 can be reinterpreted as follows.

Proposition 3.5. $\quad$ Let $\mathcal{X}$ be the germ of a formal $R$-curve at a smooth point $x$ and let $f: \mathcal{Y} \rightarrow \mathcal{X}$ be a cyclic cover of degree $p$, with $\mathcal{Y}$ normal and local. Then one can associate with $f$, canonically, simple degeneration data $\operatorname{Deg}(x) \in \operatorname{Deg}_{\mathbf{p}}$ which describes the semi-stable reduction of $\mathcal{Y}$. In other words, there exists a canonical "specialization" map $\mathrm{Sp}: H_{\mathrm{et}}^{1}(\operatorname{Spec} L, \mathbb{Z} / p \mathbb{Z}) \rightarrow \operatorname{Deg}_{\mathbf{p}}$, where $L$ is the function field of $\overline{\mathcal{X}}:=\mathcal{X} \times_{R} \bar{R}$ and $\bar{R}$ is the integral closure of $R$ in an algebraic closure of $K$.

Reciprocally, we have the following result of realization of degeneration data for such covers.

Theorem 3.6. The above specialization map $\mathrm{Sp}: H_{\mathrm{et}}^{1}(\operatorname{Spec} L, \mathbb{Z} / p \mathbb{Z}) \rightarrow$ Deg $_{\mathbf{p}}$ defined in 3.4 is surjective.

Proof. Consider simple degeneration data $\operatorname{Deg}(x) \in \mathbf{D e g}_{\mathbf{p}}$ of type $(r,(m, n))$ and rank $p$ torsor. We assume for simplicity that $\operatorname{Deg}(x)$ is $K$ degeneration data. We have to show that $\operatorname{Deg}(x)$ is associated, via the map in 
3.5 , to some cyclic $p$-cover above the formal germ of an $R$-curve at a smooth point. We only treat explicitly the case where $n=v_{K}(\lambda)$, the remaining cases are treated similarly. The proof is done by induction on the length of the tree $\Gamma$ of $\operatorname{Deg}(x)$. Assume first that the tree $\Gamma$ has minimal length and consists of one irreducible component $X:=X_{i}=\mathbb{P}_{k}^{1}$ with one marked (double) point $z$ and $r>0$ smooth distinct marked points $\left\{x_{j}:=x_{i, j}\right\}_{j=1}^{r}$. Let $\mathcal{X}$ be a proper semi-stable $R$-curve whose special fiber consists of two irreducible smooth components $X$ and $X_{1}$ of genus 0 which intersect at an ordinary double point $z$ of thickness $e=p t$ (and where the component $X$ is marked as above). Let $U:=X-\left\{z, x_{j}\right\}_{j=1}^{r}\left(\right.$ resp. $\left.U^{\prime}:=X-\{z\}\right)$, and let $\mathcal{U}$ (resp. $\mathcal{U}^{\prime}$ ) be the formal fiber of $U$ (resp. of $U^{\prime}$ ) in $\mathcal{X}$.

First, for each $j \in\{1, \ldots, r\}$ consider the formal germ $\mathcal{X}_{j}:=\operatorname{Spf} R\left[\left[T_{j}\right]\right]$ of the point $x_{j}$ in $\mathcal{X}$ and the cyclic cover $f_{j}: \mathcal{Y}_{j} \rightarrow \mathcal{X}_{j}$ of degree $p$ given by the equation $Y_{j}^{p}=T_{j}^{h_{j}}$, where $h_{j}:=h_{i, j}$ is the "residue" associated with the point $x_{j}:=x_{i, j}$ in Deg.5. Let $\bar{f}: V \rightarrow U$ be the torsor given by the data Deg.4, which is necessarily a $\mu_{p}$-torsor given by an equation $t^{p}=\bar{u}$, where $\bar{u}$ is a regular function on $U$. Let $u$ be a regular function on $\mathcal{U}$ which lifts $\bar{u}$. Then the cover $f: \mathcal{V} \rightarrow \mathcal{U}$ given by the equation $Y^{p}=u$ is a $\mu_{p}$-torsor which lifts the $\mu_{p}$-torsor $\bar{f}$. By construction, the torsor $f$ has a reduction on the formal boundary at each point $x_{j}$ of type $\left(\mu_{p}, 0, h_{i, j}\right)$, which coincides with the degeneration type of the cover $f_{j}$ above the boundary of $\mathcal{X}_{j}$. The technique of formal patching (cf. [14], 1) allows one to construct a cyclic cover $f^{\prime}: \tilde{\mathcal{V}}^{\prime} \rightarrow \tilde{\mathcal{U}}^{\prime}$ of degree $p$, where $\tilde{\mathcal{U}}^{\prime}$ is a formal affine scheme obtained by gluing $\mathcal{U}$ to the $\mathcal{X}_{j}(j \in\{1, \ldots, r\})$ (this is done by identifying the boundary of $\mathcal{X}_{j}$ with the corresponding boundary of $\mathcal{U}$ via some specific isomorphisms), which when restricted to $\mathcal{U}$ is isomorphic to $f$, and when restricted to $\mathcal{X}_{j}$ for each $j \in\{i, \ldots, r\}$ is isomorphic to $f_{j}$ (cf. loc. cit. I, and Remark 2.5). Note that in general $\tilde{\mathcal{U}}^{\prime}$ is not isomorphic to $\mathcal{U}^{\prime}$. Indeed, the identifications of formal boundaries used to construct $\tilde{\mathcal{U}}^{\prime}$ (which are specific isomorphisms (cf. [14], Remark 2.5)), need not be compatible with those which lead to the construction of $\mathcal{U}^{\prime}$ by gluing $\mathcal{U}$ to the $\mathcal{X}_{j}, j \in\{1, \ldots, r\}$.

Let $e=p t$ be the positive integer associated with the marked double point $z$ via Deg.7. We have $v_{K}(\lambda)=-m t$ by assumption. Let $\mathcal{X}_{1}^{\prime}$ be the formal fiber of $X_{1}^{\prime}:=X_{1}-\{z\}$ in $\mathcal{X}$, and let $f_{1}^{\prime}: \mathcal{Y}_{1}^{\prime} \rightarrow \mathcal{X}_{1}^{\prime}$ be the étale $\mathbb{Z} / p \mathbb{Z}$-torsor given by the equation $Y^{\prime p}=1+\lambda^{p} S^{m}$, where $S$ is a "parameter" on $\mathcal{X}_{1}^{\prime}$ which vanishes at $z$. Further, let $\mathcal{X}_{z} \simeq \operatorname{Spf} R[[S, T]] /\left(S T-\pi^{p t}\right)$ be the formal germ of $\mathcal{X}$ at the double point $z$ and consider the cover $f_{z}: \mathcal{Y}_{y} \rightarrow \mathcal{X}_{z}$ given by the equation $Y^{p}=1+\lambda^{p} S^{m}$. Then $\mathcal{Y}_{y}$ is the formal germ of a double point of thickness $t$ (cf. [14], 4.2.4, example (4)). Moreover, the cover $f_{1}^{\prime}$ (resp. $f^{\prime}$ ) 
constructed above has the same degeneration type (by construction) on the boundary corresponding to the double point $z$ as the degeneration type of the cover $f_{z}$ above the formal boundary with parameter $S$ (resp. above the formal boundary with parameter $T$ ). A second application of the formal patching technique allows one to construct a $p$-cyclic cover $g: \tilde{\mathcal{Y}} \rightarrow \tilde{\mathcal{X}}$, where $\tilde{\mathcal{X}}$ is a formal semi-stable $R$-curve whose special fiber is isomorphic to $\mathcal{X}_{k}$, which is obtained by gluing $\tilde{\mathcal{U}}^{\prime}, \mathcal{X}_{1}^{\prime}$ and $\mathcal{X}_{z}$ (by some specific identification of the boundaries). Further, $g$ restricted to $\tilde{\mathcal{U}}^{\prime}$ (resp. $\mathcal{X}_{1}^{\prime}, \mathcal{X}_{z}$ ) is isomorphic to $f^{\prime}$ (resp. $f_{1}^{\prime}, f_{z}$ ). Note that, in general, $\tilde{\mathcal{X}}$ is not isomorphic to $\mathcal{X}$. Let $\tilde{\mathcal{X}}^{\prime}$ be the $R$-curve obtained by contracting the irreducible component $X$ in $\tilde{\mathcal{X}}$. We denote by $\tilde{x}$ the image of the double point $z$ in $\tilde{\mathcal{X}}$. The cover $g: \tilde{\mathcal{Y}} \rightarrow \tilde{\mathcal{X}}$ induces canonically a $p$-cyclic cover $\tilde{f}: \tilde{\mathcal{Y}}^{\prime} \rightarrow \tilde{\mathcal{X}}^{\prime}$ above $\tilde{\mathcal{X}}^{\prime}$. Let $\mathcal{X}_{\tilde{x}} \simeq R[[S]]$ be the formal germ of $\tilde{\mathcal{X}}^{\prime}$ at the smooth point $\tilde{x}$. Then $\tilde{f}$ induces canonically a $p$-cyclic cover $f_{\tilde{x}}: \mathcal{Y}_{\tilde{y}} \rightarrow \mathcal{X}_{\tilde{x}}$ where $\tilde{y}$ is the closed point of $\tilde{\mathcal{Y}}$ above $\tilde{x}$. Now it is easy to see that the degeneration data associated with $f_{\tilde{x}}$, via 3.5 , is isomorphic to the degeneration data $\operatorname{Deg}(x)$ we started with. Finally, the proof in the general case is very similar and is left to the reader. The only modification is that one has to consider $p$-cyclic covers $f_{i, j}: \mathcal{Y}_{i, j} \rightarrow \mathcal{X}_{i, j}$ above the formal germs $\mathcal{X}_{i, j}:=\operatorname{Spf} R\left[\left[T_{i, j}\right]\right]$. These are obtained by the induction hypothesis as realization of the degeneration data, induced by $\operatorname{Deg}(x)$, on the subtrees $\Gamma_{i, j}$ of $\Gamma$ which start from the edge $x_{i}$ in the direction of the ends of $\Gamma$, and which clearly have length smaller than the length of $\Gamma$.

Remarks 3.7. 1. The surjectivity result in 3.6 was shown in [5] in the case of simple degeneration data $\operatorname{Deg}(x)$ of arithmetic genus $g_{x}=0$, in the framework of automorhisms of order $p$ of open discs and annuli.

2. Let $f: \mathcal{Y} \rightarrow \mathcal{X}$ be a Galois cover of degree $p$ above the formal germ $\mathcal{X} \simeq \operatorname{Spf} R[[T]]$ of a smooth point $x$. In [12] Raynaud proved that if $f$ is étale above the generic fiber $\mathcal{X}_{K}$ of $\mathcal{X}$ then the special fiber $\mathcal{Y}_{k}$ of $\mathcal{Y}$ is unibranch, and the configuration of the special fiber of a semi-stable model of $\mathcal{Y}$ is treelike. If $f$ is not étale above $\mathcal{X}_{K}$ then this is no longer true, in general. Indeed, consider the following example. Let $\operatorname{Deg}(x)$ be $K$-simple degeneration data (of rank $p$ torsor) of type $(2,(0,-1))$, which consist of a graph $\Gamma$ with two vertices $X_{1}$ and $X_{2}$ linked by a unique edge $z$ and with given marked smooth points $x=x_{1}$ on $X_{1}$, and $x_{2}$ on $X_{2}$. Further, assume that are given (by Deg.4) étale $\mathbb{Z} / p \mathbb{Z}$-torsors $f_{1}: V_{1} \rightarrow U_{1}:=X_{1}-\left\{x_{1}\right\}$ with conductor $m_{1}=1$ at $x_{1}$, and $f_{2}: V_{2} \rightarrow U_{2}:=X_{2}-\left\{x_{2}\right\}$, with conductor $m_{2}=1$ at $x_{2}$. Then, it follows from 3.6 that there exists (after perhaps a finite extension of $R$ ) a Galois cover of degree $p f: \mathcal{Y} \rightarrow \mathcal{X}$ whose semi-stable reduction gives rise to the degeneration 
data $\operatorname{Deg}(x)$. Let $\tilde{\mathcal{Y}}$ be the minimal semi-stable model of $\mathcal{Y}$, which is defined over $R$, and let $\tilde{\mathcal{X}}$ be its quotient by the Galois group of $f$, which is a semi-stable model of $\mathcal{X}$. By construction, the special fiber $\tilde{\mathcal{X}}_{k}$ of $\tilde{\mathcal{X}}$ consists of the graph $\Gamma$ which is linked to the germ at the smooth point $x$. The cover $\tilde{f}: \tilde{\mathcal{Y}} \rightarrow \tilde{\mathcal{X}}$ between semi-stable models is, by construction, étale above the double point $z$. Thus, the special fiber $\tilde{\mathcal{Y}}_{k}$ of $\tilde{\mathcal{Y}}$ consists necessarily of two projective lines $Y_{1}, Y_{2}$, which are the normalizations of $V_{1}, V_{2}$, and which intersect at $p$-double points which are the pre-images of $z$. In particular, there are $p-1$ cycles in the configuration of $\tilde{\mathcal{Y}}_{k}$.

3. Using the results of Sections 1 and 2 one can also define in the same way as in 3.4 the set of isomorphism classes of "double" degeneration data associated with the minimal semi-stable model of a $p$-cyclic cover $f: \mathcal{Y} \rightarrow \mathcal{X}$ above the formal germ $\mathcal{X}$ of a formal $R$-curve at an ordinary double point. Moreover, one can prove, in a similar way as in 3.6, a result of realization for such degeneration data.

\section{§4. Semi-Stable Reduction of Cyclic $p$-Covers above Proper Curves in Mixed Characteristic}

In what follows we use the same notation as in Section 3. Our aim in this section is to describe the "degeneration data" that arise from the semi-stable reduction of Galois covers of degree $p$ above proper smooth $R$-curves, and prove a realization theorem for such data.

4.1. Let $\mathcal{X}$ be a formal, smooth and proper $R$-curve, and let $f: \mathcal{Y} \rightarrow \mathcal{X}$ be a Galois cover with group $G \simeq \mathbb{Z} / p \mathbb{Z}$, such that $\mathcal{Y}$ is normal. We denote by $X$ the special fiber $\mathcal{X}_{k}:=\mathcal{X} \times_{R} k$ of $\mathcal{X}$. Let $R^{\prime}, K^{\prime}, \mathcal{X}^{\prime}, \mathcal{Y}^{\prime}, \tilde{\mathcal{X}}$, and $\tilde{\mathcal{Y}}$ be as 3.1. Recall that we have the following commutative diagram:

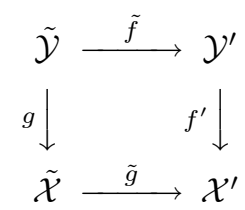

4.2. We will use the same notation as in 3.1 and 4.1. Let $R^{\prime}$ be a finite extension of $R$ as in 3.1 and let $\pi^{\prime}$ be a uniformizer of $R^{\prime}$. Below we exhibit the degeneration data associated with the minimal semi-stable reduction $\tilde{\mathcal{Y}}$ of $\mathcal{Y}$. 
Deg.1. Let $\eta$ be the generic point of the special fiber of $\mathcal{X}^{\prime}$ and let $\mathcal{X}_{\eta}^{\prime}$ be the formal germ of $\mathcal{X}^{\prime}$ at $\eta$. Consider the following cartesian diagram:

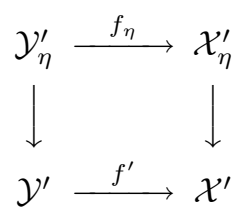

Then $f_{\eta}: \mathcal{Y}_{\eta}^{\prime} \rightarrow \mathcal{X}_{\eta}^{\prime}$ is a torsor under a finite commutative and flat $R^{\prime}$-group scheme $G_{R^{\prime}}$ of rank $p$, which is either isomorphic to $\mu_{p}$, or to the group scheme $\mathcal{H}_{n, R^{\prime}}$ (cf. 1.1) for some integer $0<n \leq v_{K^{\prime}}(\lambda)$, as follows from 1.3. We denote by $\left(G_{k}, m, h\right)$ the degeneration type of the torsor $f_{\eta}$ (cf. 1.7), which is canonically associated with $f$. Note that $G_{k}$ is isomorphic to the special fiber of the group scheme $G_{R^{\prime}}$.

Deg.2. Let $\left\{x_{i}\right\}_{i \in I^{\prime}}$ be the finite set of closed points of $X$ in which some branched points of the morphism $f_{K^{\prime}}^{\prime}: \mathcal{Y}_{K^{\prime}}^{\prime} \rightarrow \mathcal{X}_{K^{\prime}}^{\prime}$ specialize. We denote this set by $B_{k}$. Let $U^{\prime}:=X-B_{k}$ and let $\mathcal{U}^{\prime}$ be the formal fiber of $U^{\prime}$ in $\mathcal{X}^{\prime}$. Then the restriction $f^{\prime \prime}: \mathcal{V}^{\prime} \rightarrow \mathcal{U}^{\prime}$ of $f^{\prime}$ to $\mathcal{U}^{\prime}$ is a torsor under a finite and flat $R$-group scheme of rank $p$ (as follows from 1.3) which is necessarily isomorphic to $G_{R^{\prime}}$. If the torsor $f^{\prime \prime}$ is radicial let $\omega$ be the associated differential form (cf. [13], 1) and let $Z_{K}$ be the set of zeros of $\omega$. Let $\operatorname{Crit}(f)=B_{k}$ if $f^{\prime \prime}$ is étale (resp. Crit $(f)=B_{k} \cup Z_{k}$ if $f^{\prime \prime}$ is radicial) and call this the set of critical points of $f$. If $y \in \mathcal{Y}$ is a singular point then $x=f(y) \in \operatorname{Crit}(f)$ necessarily.

Deg.3. Let $\operatorname{Crit}(f)=\left\{x_{i}\right\}_{i \in I}$. For each $i \in I$, let $\mathcal{X}_{i}^{\prime}$ be the formal fiber of $x_{i}$ in $\mathcal{X}^{\prime}$ and let $f_{i}^{\prime}: \mathcal{Y}_{i}^{\prime} \rightarrow \mathcal{X}_{i}^{\prime}$ be the cover induced by $f^{\prime}$. Let $\left(G_{i, k}, m_{i}, h_{i}\right)$ be the degeneration type of $f_{i}^{\prime}$ on the boundary (cf. 1.7). Then necessarily all the group schemes $G_{i, k}$ are isomorphic to the special fiber $G_{k}$ of the group scheme $G_{R^{\prime}}$ in Deg.1. Further, we have $\sum_{i \in I} h_{i}=0$ as follows from the residue theorem.

Deg.4. For each $i \in I$, the Galois cover $f_{i}^{\prime}: \mathcal{Y}_{i}^{\prime} \rightarrow \mathcal{X}_{i}^{\prime}$ gives rise, via 3.5 , to $K^{\prime}$-simple degeneration data $\operatorname{Deg}\left(x_{i}\right)$ of type $\left(r_{i},\left(n_{i}, m_{i}\right)\right)$, and rank $p$ torsor where $r_{i}$ is the number of branched points which specialize in $x_{i}$, and $n_{i}$ necessarily equals either 0 if $G_{R^{\prime}} \simeq \mu_{p}$ or $n$ if $G_{R^{\prime}} \simeq \mathcal{H}_{n, R^{\prime}}$.

The above considerations lead naturally to the following abstract geometric and combinatorial definition of degeneration data.

Definition 4.3. Smooth $K^{\prime}$-degeneration data $\operatorname{Deg}(\mathrm{X})$ of rank $p$ torsor consist of the following data:

Deg.1. $K^{\prime}$ is a finite extension of $K$. Further, $X$ is a proper and smooth $k$ curve endowed with a finite set $B_{k}$ of closed (mutually distinct) marked points. 
Let $U:=X-B_{k}$.

Deg.2. $\bar{f}: V \rightarrow U$ is a torsor under a finite and flat $k$-group scheme $G_{k}$ of rank $p$ and $0 \leq n \leq v_{K^{\prime}}(\lambda)$ is an integer which equals 0 (resp. equals $v_{K^{\prime}}(\lambda)$ ) if and only if $G_{k}$ is of multiplicative type (resp. if and only if $G_{k}$ is étale).

Deg.3. Let $\operatorname{Crit}(\bar{f})=\left\{x_{i}\right\}_{i \in I}$ be the set $B_{k}$ if $\bar{f}$ is étale $(\operatorname{resp} . \operatorname{Crit}(\bar{f})=$ $B_{k} \cup Z_{k}$ if $\bar{f}$ is radicial, where $Z_{k}$ is the set of zeros of the corresponding differential form). For each $i \in I$, is given $K^{\prime}$-simple degeneration data $\operatorname{Deg}\left(x_{i}\right)$ of type $\left(r_{i},\left(n, m_{i}\right)\right)$ and rank $p$ torsor, where $m_{i}$ be the conductor of the above torsor $\bar{f}$ at the point $x_{i}$ (cf. [13], I).

There is a natural notion of isomorphism of smooth degeneration data of rank $p$ torsor relative to a given finite extension $K^{\prime}$ of $K$ and associated with a smooth and proper $k$-curve $X$. We will denote by $\mathbf{D E G}_{\mathbf{p}}(\mathbf{X})$ the set of isomorphism classes of smooth degeneration data of rank $p$ torsor associated with $X$. The above discussion in 4.2 can be reinterpreted as follows.

Proposition 4.4. $\quad$ Let $\mathcal{X}$ be a formal proper and smooth $R$-curve with special fibre $\mathcal{X}_{k}:=\mathcal{X} \times_{R} k$, and let $f: \mathcal{Y} \rightarrow \mathcal{X}$ be a cyclic cover of degree $p$ with $\mathcal{Y}$ normal. Then one can associate with $f$, canonically, a smooth degeneration data $\operatorname{Deg}\left(\mathcal{X}_{k}\right) \in \mathbf{D E G}_{\mathbf{p}}\left(\mathcal{X}_{\mathbf{k}}\right)$ which describes the semi-stable reduction of $\mathcal{Y}$. In other words there exists a canonical "specialization" map $\mathrm{Sp}$ : $H_{\mathrm{et}}^{1}(\operatorname{Spec} L, \mathbb{Z} / p \mathbb{Z}) \rightarrow \mathbf{D E G}_{\mathbf{p}}\left(\mathcal{X}_{\mathbf{k}}\right)$, where $L$ is the function field of $\overline{\mathcal{X}}:=\mathcal{X} \times{ }_{R} \bar{R}$ and $\bar{R}$ is the integral closure of $R$ in an algebraic closure of $K$.

Reciprocally, we have the following result of realization of degeneration data for such covers.

Theorem 4.5. Let $\operatorname{Deg}(X) \in \mathbf{D E G}_{\mathbf{p}}(\mathbf{X})$ be a smooth degeneration data of rank $p$ torsor associated with the proper and smooth $k$-curve $X$. Then, there exists a smooth formal and proper $R$-curve $\mathcal{X}^{\prime}$, with special fibre $\mathcal{X}_{k}^{\prime}:=$ $\mathcal{X}^{\prime} \times_{R} k$ isomorphic to $X$, and such that $\operatorname{Deg}(X)$ is in the image of the specialization map Sp : $H_{\text {et }}^{1}(\operatorname{Spec} L, \mathbb{Z} / p \mathbb{Z}) \rightarrow \mathbf{D E G}_{\mathbf{p}}(\mathbf{X})$ defined in 4.4 , where $L$ is the function field of $\mathcal{X}^{\prime} \times_{R} \bar{R}$ and $\bar{R}$ is the integral closure of $R$ in an algebraic closure of $K$.

Proof. Consider smooth degeneration data $\operatorname{Deg}(X) \in \mathbf{D E G}_{\mathbf{p}}(\mathbf{X})$. We assume for simplicity that $\operatorname{Deg}(X)$ is $K$-degeneration data. We have to show that $\operatorname{Deg}(X)$ is associated, via the map in 4.4 , with some cyclic cover of degree $p$ above a formal proper and smooth $R$-curve $\mathcal{X}^{\prime}$. We only treat explicitly the case where $n=v_{K}(\lambda)$ (i.e. the torsor $\bar{f}$ in Deg.2 is étale), the remaining cases are treated similarly. Let $\mathcal{X}$ be a formal smooth and proper $R$-curve whose 
special fibre $\mathcal{X}_{k}$ is isomorphic to $X$. Let $\mathcal{U}$ be the formal fibre of $U$ in $\mathcal{X}$, and for $i \in I$, let $\mathcal{X}_{i}$ be the formal fibre of $x_{i}$ in $\mathcal{X}$. The étale torsor $\bar{f}$ given by Deg.2 can be lifted to an étale torsor $f: \mathcal{V} \rightarrow \mathcal{U}$ by the theorems of lifting of étale covers (one can in this specific situation write down an explicit lifting). Also, for $i \in I$, let $f_{i}: \mathcal{Y}_{i} \rightarrow \mathcal{X}_{i}$ be a Galois cover of degree $p$ which is a realization (as in 3.6) of the degeneration data $\operatorname{Deg}\left(x_{i}\right)$ given by Deg.3, and which is of type $\left(r_{i},\left(v_{K}(\lambda), m_{i}\right)\right)$, where $m_{i}$ is the Hasse-conductor of $\bar{f}$ at $x_{i}$. By construction, the covers $f$ and $f_{i}$ coincide on the formal boundaries at the points $x_{i}$. The technique of formal patching (cf. [14], 1) allows one to construct a cyclic cover $f^{\prime}: \mathcal{Y}^{\prime} \rightarrow \mathcal{X}^{\prime}$ of degree $p$, where $\mathcal{X}^{\prime}$ is a proper and smooth formal curve which is obtained by glueing $\mathcal{U}$ to the $\mathcal{X}_{i}(j \in\{1, \ldots, r\})$ by identifying the boundaries corresponding to each point $x_{i}$ via some specific isomorphisms. Further, $f^{\prime}$ restricted to $\mathcal{U}$ is isomorphic to $f$, and $f^{\prime}$ restricted to $\mathcal{X}_{i}$, for each $i \in I$, is isomorphic to $f_{i}$ (cf. loc. cit.). Note that in general $\mathcal{X}^{\prime}$ is not isomorphic to $\mathcal{X}$. Indeed, the identifications of formal boundaries used to construct $\mathcal{X}^{\prime}$ (which are specific isomorphisms) need not be compatible with those which lead to the construction of $\mathcal{X}$ by gluing $\mathcal{U}$ to the $\mathcal{X}_{i}$.

Example 4.6. Assume $p>2$. Let $X$ be a proper and smooth $k$-curve of genus $g \geq 2$, which we assume to be generic (cf. [12], p. 193). Let $\mathcal{X}$ be a smooth and proper $R$-curve with special fiber $\mathcal{X}_{k}$ which is isomorphic to $X$. Let $f: \mathcal{Y} \rightarrow \mathcal{X}$ be a Galois cover of degree $p$ which is étale above the generic fiber $\mathcal{X}_{K}$ of $\mathcal{X}$ and with $\mathcal{Y}_{k}$ reduced. Then $f$ is a torsor under a finite, flat, and commutative group scheme of rank $p$, as follows from 1.3. Further, the following two cases occur. Either $f$ is an étale torsor, in which case $f$ is smooth. Or $f$ is a $\mu_{p}$-torsor (note that because $X$ is ordinary it admits no non-trivial $\alpha_{p}$-torsors). In the latter case $f$ induces a $\mu_{p}$-torsor $f_{k}: Y \rightarrow X$ on the level of special fibers, where $Y:=\mathcal{Y}_{k}$ is the special fiber of $\mathcal{Y}$. Let $\omega$ be the logarithmic differential form associated with $f_{k}$ (cf. [13], 1.4). Then the set of critical points $\operatorname{Crit}\left(f_{k}\right)$ of $f_{k}$ (cf. 4.2, Deg.3) coincides with the set of zeros of $\omega$ (cf. [13], 1.4). It is shown in [12], loc. cit., that $\omega$ has $2 g-2$ simple zeros $\left\{x_{i}\right\}_{i=1}^{2 g-2}$. The degeneration type of $f$ on the boundary of the formal fiber at each point $x_{i}$ is $\left(\mu_{p},-2,0\right)$. Let $\mathcal{X}_{i}$ be the formal germ of $\mathcal{X}$ at the point $x_{i}$, and let $f_{i}: \mathcal{Y}_{i} \rightarrow \mathcal{X}_{i}$ be the cover induced by $f$. Then to $f_{i}$ is associated, via 3.5 , simple degeneration data $\operatorname{Deg}\left(x_{i}\right)$ of type $\left(0,\left(v_{K}(\lambda), 2\right)\right)$, which consist necessarily of a tree $\Gamma_{i}$ with one vertex $X_{i}$, no edges, and one marked (double) point $x_{i}$ on $X_{i}$. Moreover, there is an étale torsor $V_{i} \rightarrow U_{i}:=X_{i}-\left\{x_{i}\right\}$ with Hasse conductor 2 at the point $x_{i}$. Let $\tilde{\mathcal{Y}}$ be the minimal semi-stable model of $\mathcal{Y}$, which we assume to be defined over $R$ for simplicity. Then, the special fiber $\tilde{\mathcal{Y}}_{k}$ of $\tilde{\mathcal{Y}}$ consists of a component 
$\tilde{Y}$, which is the normalization of $Y$, and $2 g-2$ double points $\left\{\tilde{y}_{i}\right\}_{i=1}^{2 g-2}$ (which are the pre-images of the critical points of $f_{k}$ ) supported by $\tilde{\mathcal{Y}}_{k}$, and each point $\tilde{y}_{i}$ is supported by another component $Y_{i}$, which is the normalization of $V_{i}$. In particular, the genus of $Y_{i}$ equals $(p-1) / 2$. Reciprocally, Theorem 4.5 translates in this example as follows. Given $(K-)$ simple degeneration data $\operatorname{Deg}\left(x_{i}\right)$ of type $\left(0,\left(v_{K}(\lambda), 2\right)\right)$ for each $i \in\{1, \ldots, 2 g-2\}$ one can find a smooth and proper $R$-curve $\mathcal{X}^{\prime}$, with special fiber $\mathcal{X}_{k}^{\prime}$ isomorphic to $X$, and a Galois cover of degree $p: f^{\prime}: \mathcal{Y}^{\prime} \rightarrow \mathcal{X}^{\prime}$, such that the simple degeneration data associated with each point $x_{i}$, and which describes the minimal semi-stable model of $\mathcal{Y}^{\prime}$, is isomorphic to the above given data $\operatorname{Deg}\left(x_{i}\right)$.

The technique of formal patching used in the proof of 4.5 can be used to prove the following result.

Theorem 4.7. Let $X$ be a smooth and proper $k$-curve and let $f: Y \rightarrow$ $X$ be a torsor under a finite and flat $k$-group scheme $G_{k}$ of rank $p$. Then there exists, after possibly a finite extension of $R$, a smooth and proper $R$-curve $\mathcal{X}$ with special fiber isomorphic to $X$ and a torsor $\tilde{f}: \mathcal{Y} \rightarrow \mathcal{X}$ under an $R$-group scheme $G_{R}$, which is commutative finite and flat of rank $p$, such that the torsor $\tilde{f}_{k}: \mathcal{Y}_{k} \rightarrow \mathcal{X}_{k}$ induced by $\tilde{f}$ on the level of special fibers is isomorphic to $f$. In other words the torsor $\tilde{f}$ lifts $f$. In particular, $G_{k}$ is isomorphic to the special fiber of $G_{R}$.

Proof. When $f$ is an étale torsor 4.7 is a consequence of the theorems of lifting of étale covers (cf. [16]). Moreover, in this case one can specify the lifting $\mathcal{X}$ of the curve $X$. The same is true when $f$ is a torsor of multiplicative type (cf. [13], 4.5). So it remains to treat the case where $G_{k} \simeq \alpha_{p}$. By the formal GAGA theorems it suffices to realize a lifting of $f$ over a formal proper and smooth $R$-curve $\mathcal{X}$. Let $\left\{y_{i}\right\}_{i \in I}$ be the finite set of singular points in $Y$ and let $\left\{x_{i}\right\}_{i \in I}$ be the set of their images in $X$, which we call the set of critical points of the torsor $f$. For $i \in I$, let $m_{i}>1$ be the conductor of the torsor $f$ at the point $x_{i}$ (cf. [13], 1.5). The arithmetic genus $g_{y}$ of $y$ equals $(m-1)(p-1) / 2$.

Let $U:=X-\left\{x_{i}\right\}_{i \in I}$ and let $\mathcal{U}$ be a formal affine scheme whose special fiber equals $U$. Also, for $i \in I$, let $\mathcal{X}_{i} \simeq \operatorname{Spf} R\left[\left[T_{i}\right]\right]$ be the formal germ of an $R$-curve at a smooth point $x_{i}$. The restriction $f^{\prime}: V \rightarrow U$ of $f$ to $U$ is an $\alpha_{p}$ torsor given by an equation $y^{p}=\bar{u}$ where $\bar{u}$ is a regular function on $U$. Let $\omega$ be the differential form $d \bar{u}$. Then $\omega$ is a global differential form on $X$ : it is the differential form associated with the torsor $f$. Moreover, the conductor $m_{i}$ equals $\operatorname{ord}_{x_{i}}(\omega)+1$ (cf. [13], 5.1). 
Let $0<n<v_{K}(\lambda)$ be an integer (we may first have to perform a finite extension of $R$ so that this condition is satisfied). Let $\tilde{f}: \mathcal{V} \rightarrow \mathcal{U}$ be the $\mathcal{H}_{n, R^{-}}$ torsor given by an equation $Y^{p}=1+\pi^{n p} u$, where $u$ is a regular function on $\mathcal{U}$

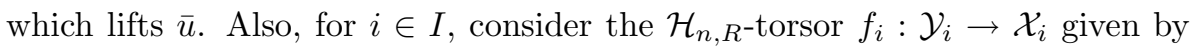
an equation $Y^{p}=1+\pi^{n p} T_{i}^{m_{i}}$, which has a reduction on the boundary of type $\left(\alpha_{p},-m_{i}, 0\right)$. By construction, this is the same reduction type as that of the torsor $\tilde{f}$ on the formal boundary corresponding to the point $x_{i}$. The technique of formal patching (cf. [14], 1) allows one to construct an $\mathcal{H}_{n, R^{-}}$-torsor $f^{\prime}: \mathcal{Y} \rightarrow \mathcal{X}$, where $\mathcal{X}$ is a proper and smooth formal curve $(\mathcal{X}$ is obtained by gluing $\mathcal{U}$ to the $\mathcal{X}_{i}(i \in I)$, by identifying the boundaries corresponding to the points $\left\{x_{i}\right\}_{i \in I}$ via some specific isomorphisms) which when restricted to $\mathcal{U}$ is isomorphic to $\tilde{f}$, and when restricted to $\mathcal{X}_{i}$, for each $i \in I$, is isomorphic to $f_{i}$ (cf. loc. cit.). In particular, the special fiber of $\mathcal{X}$ is isomorphic to $X$.

Note that, in general, if $\mathcal{X}^{\prime}$ is another formal curve which lifts $X$, then we can not construct a lifting $f^{\prime}$ of $f$ (as above) above the curve $\mathcal{X}^{\prime}$, even if the formal fiber of $U$ in $\mathcal{X}^{\prime}$ is isomorphic to $\mathcal{U}$. Indeed, the identification of formal boundaries used to construct $\mathcal{X}$ (which are specific isomorphisms) need not be compatible with those which lead to the construction of $\mathcal{X}^{\prime}$ by gluing $\mathcal{U}$ to the $\mathcal{X}_{i}$

Remark 4.8. 1. Theorem 4.5 is stronger than 4.7. Indeed, a translation of 4.6 in the language of 4.5 leads not only to the lifting of the torsor $f$ but to a lifting with specified simple degeneration data at the critical points.

2. Theorem 4.7 is also proved in [1] using different methods.

3. The same proof as in 4.7 shows that it is possible to lift (as above) $\alpha_{p^{n}}$-torsors $f: Y \rightarrow X$ above a proper and smooth $k$-curve $X$.

\section{Acknowledgement}

The author holds an EPSRC advanced research fellowship GR/R75861/02 and would like very much to thank EPSRC for its support.

\section{References}

[1] F. Andreatta and G. Gasbarri, Deformation of torsors under group schemes of order $p$. 2003, Preprint.

[2] S. Bosch and W. Lütkebohmert, Formal and rigid geometry. I. Rigid spaces, Math. Ann. 295 (1993), no. 2, 291-317.

[3] I. I. Bouw and S. Wewers, The local lifting problem for dihedral groups, Duke Math. J. 134 (2006), no. 3, 421-452.

[4] P. Deligne and D. Mumford, The irreducibility of the space of curves of given genus, Inst. Hautes Études Sci. Publ. Math. No. 36 (1969), 75-109. 
[5] Y. Henrio, Arbres de Hurwitz et automorphismes d'ordre $p$ des disques et couronnes $p$-adiques. Thèse de Doctorat, (1999), Université Bordeaux I, France.

[6] C. Lehr, Reduction of $p$-cyclic covers of the projective line, Manuscripta Math. 106 (2001), no. 2, 151-175.

[7] C. Lehr and M. Matignon, Wild monodromy and automorphisms of curves, Duke Math. J. 135 (2006), no. 3, 569-586.

[8] Q. Liu, Stable reduction of finite covers of curves, Compos. Math. 142 (2006), no. 1, 101-118.

[9] M. Matignon, Vers un algorithme pour la réduction stable des revêtements $p$-cycliques de la droite projective sur un corps p-adique, Math. Ann. 325 (2003), no. 2, 323-354.

[10] S. Maugeais, Relèvement des revêtements $p$-cycliques des courbes rationnelles semistables, Math. Ann. 327 (2003), no. 2, 365-393.

[11] T. Sekiguchi, F. Oort and N. Suwa, On the deformation of Artin-Schreier to Kummer, Ann. Sci. École Norm. Sup. (4) 22 (1989), no. 3, 345-375.

[12] M. Raynaud, p-groupes et réduction semi-stable des courbes, in The Grothendieck Festschrift, Vol. III, 179-197, Progr. Math., 88, Birkhäuser, Boston, Boston, MA.

[13] M. Saïdi, Torsors under finite and flat group schemes of rank $p$ with Galois action, Math. Z. 245 (2003), no. 4, 695-710.

[14] Wild ramification and a vanishing cycles formula, J. Algebra 273 (2004), no. 1, $108-128$.

$[15]+$ On the degeneration of étale $\mathbb{Z} / p \mathbb{Z}$ and $\mathbb{Z} / p^{2} \mathbb{Z}$-torsors in equal characteristic $p>0$. Hiroshima Math. J., to appear.

[16] A. Grothendieck, Revêtements étales et groupe fondamental, Lecture Notes in Math., 224, Springer, Berlin, 1971. 